\title{
Epidural hematoma after thoracic epidural analgesia in a patient treated with ketorolac, mefenamic acid, and naftazone
} -a case report-

\author{
Dae Geun Jeon, Jae Gyok Song, Seok-Kon Kim, and Juri Kim \\ Department of Anesthesiology and Pain Medicine, Dankook University College of Medicine, Cheonan, Korea
}

\begin{abstract}
A 26-year-old male undergoing thoracotomy and bleeding control received a preoperative thoracic epidural for postoperative analgesia. On the fifth postoperative day, paralysis of both lower limbs occurred and urgent magnetic resonance imaging showed massive anterior epidural hematoma. During laminectomy and decompression, platelet dysfunction was diagnosed and preoperative non-steroidal anti-inflammatory drugs medications were supposed to the cause of platelet dysfunction. After infusion of ten units of platelet concentrate, coagulopathy was improved. We should be more careful to drugs with antiplatelet effect when using regional analgesia. (Korean J Anesthesiol 2014; 66: 240-243)
\end{abstract}

Key Words: Epidural hematoma, Ketorolac, Mefenamic acid, Naftazone, Platelet function.

Spinal epidural hematoma is a rare complication of epidural anesthesia or analgesia with an estimated incidence of $1: 190,000$ that have potentially devastating consequences [1]. The relationship between spinal epidural hematoma and anticoagulation is well documented [2]. Nowadays many people are taking drugs like non-steroidal anti-inflammatory drugs (NSAIDs), herbal medications or foods which can induce platelet dysfunction but the risk of them is not well known [3,4]. We report here a case of spinal epidural hematoma in a 26-year-old male patient with no history of coagulopathy who received naftazone, mefenamic acid and ketorolac.

\section{Case Report}

A 26-year-old male (height: $174 \mathrm{~cm}$, weight, $72 \mathrm{~kg}$, American Society of Anesthesiologists physical status class I) was scheduled for wedge resection of bullae and bleeding control. His past medical history was negative for cardio-respiratory diseases, diabetes mellitus, bleeding diathesis, or easy bruising. The patient had received medications for hemorrhoid for six days until the day before admission. Physical examination and laboratory findings were unremarkable.

He referred from other hospital to our emergency room to treat hemo-pneumothorax with massive bleeding. When he

Received: November 23, 2012. Revised: 1st, November 31, 2012; 2nd, January 24, 2013; 3rd, March 5, 2013. Accepted: March 7, 2013.

Corresponding author: Jae Gyok Song, M.D., Department of Anesthesiology and Pain Medicine, Dankook University College of Medicine, 16-5, Anseo-dong, Cheonan 330-715, Korea. Tel: 82-41-550-6819, Fax: 82-41-551-9330, E-mail: codename-jack@hanmail.net

(c) This is an open-access article distributed under the terms of the Creative Commons Attribution Non-Commercial License (http:// creativecommons.org/licenses/by-nc/3.0/), which permits unrestricted non-commercial use, distribution, and reproduction in any medium, provided the original work is properly cited. 
admitted to our hospital, approximately $400 \mathrm{ml}$ of bloody fluid was drained from the chest drainage tube, and his hemoglobin (Hb) was11.0 mg/dl. Two units of packed red blood cells (PRBC) were infused to treat tachycardia (95 beats/min) with mild hypotension $(95 / 50 \mathrm{mmHg})$. His admission coagulation profiles were within normal limit (prothrombin time (PT); 13.0 seconds, International normalized ratio (INR); 1.12 , activated partial thromboplastin time (aPTT); 24.7 seconds, platelet count; $245,000 / \mathrm{mm}^{3}$ ). The patient's bleeding time was not checked.

Two hours later, his lab findings were $\mathrm{Hb} 10.5 \mathrm{mg} / \mathrm{dl}$, Hct $30.4 \%$, and platelet count $220,000 \mathrm{~mm}^{3}$. One unit of PRBC and three units of fresh frozen plasma (FFP) were infused. Eight hours after admission, more than $400 \mathrm{ml}$ of bloody fluid was drained again, and his lab findings were $\mathrm{Hb} 11.5$, Hct 33.6, and platelet count was $158,000 \mathrm{~mm}^{3}$. Thirteen hours after admission, he was transferred to operating room.

A thoracic epidural catheter at the T5-T6 level was inserted through a 17 gauge Tuohy needle using loss of resistance to air technique. First attempt at T6-T7 level, inadvertent dural puncture occurred and blood tinged CSF was seen in the syringe. Second attempt at T5-6 level was performed uneventfully. No blood or paresthesia was noted during the successful needle and catheter insertion. A test dose of $2 \mathrm{ml}$ of $2 \%$ lidocaine and $5 \mu \mathrm{g}$ epinephrine was injected through the epidural catheter and hypaesthesia was confirmed at T4-6 dermatome with a cold alcohol swab. There was no specific symptom related to epidural analgesia during the surgery.

Bullectomy, hematoma evacuation and bleeding control was conducted under general anesthesia. The operation time was 2 hours and 40 minutes and the total infused fluids were crystalloid $900 \mathrm{ml}$, colloids $400 \mathrm{ml}$, and PRBC 2 units. Following operation, the hemoglobin level was $11.6 \mathrm{mg} / \mathrm{dl}$, and the platelet count was $175,000 / \mathrm{mm}^{3}$. Epidural patient controlled analgesia (PCEA) with $0.2 \%$ ropivacaine with fentanyl $3 \mu \mathrm{g} / \mathrm{ml}$ was started after the operation.

On the first postoperative day (POD 1) oral mefenamic acid $250 \mathrm{mg}$, three times a day was started to treat mild fever. On POD2 the patient complained of headache and oral acetaminophen 1,300 mg was given two times a day. PCEA infusion was stopped. On POD3 at 6:00 AM, the patient complained of headache, neck stiffness, dizziness, and nausea and vomiting during ambulation. These symptoms were aggravated with upright
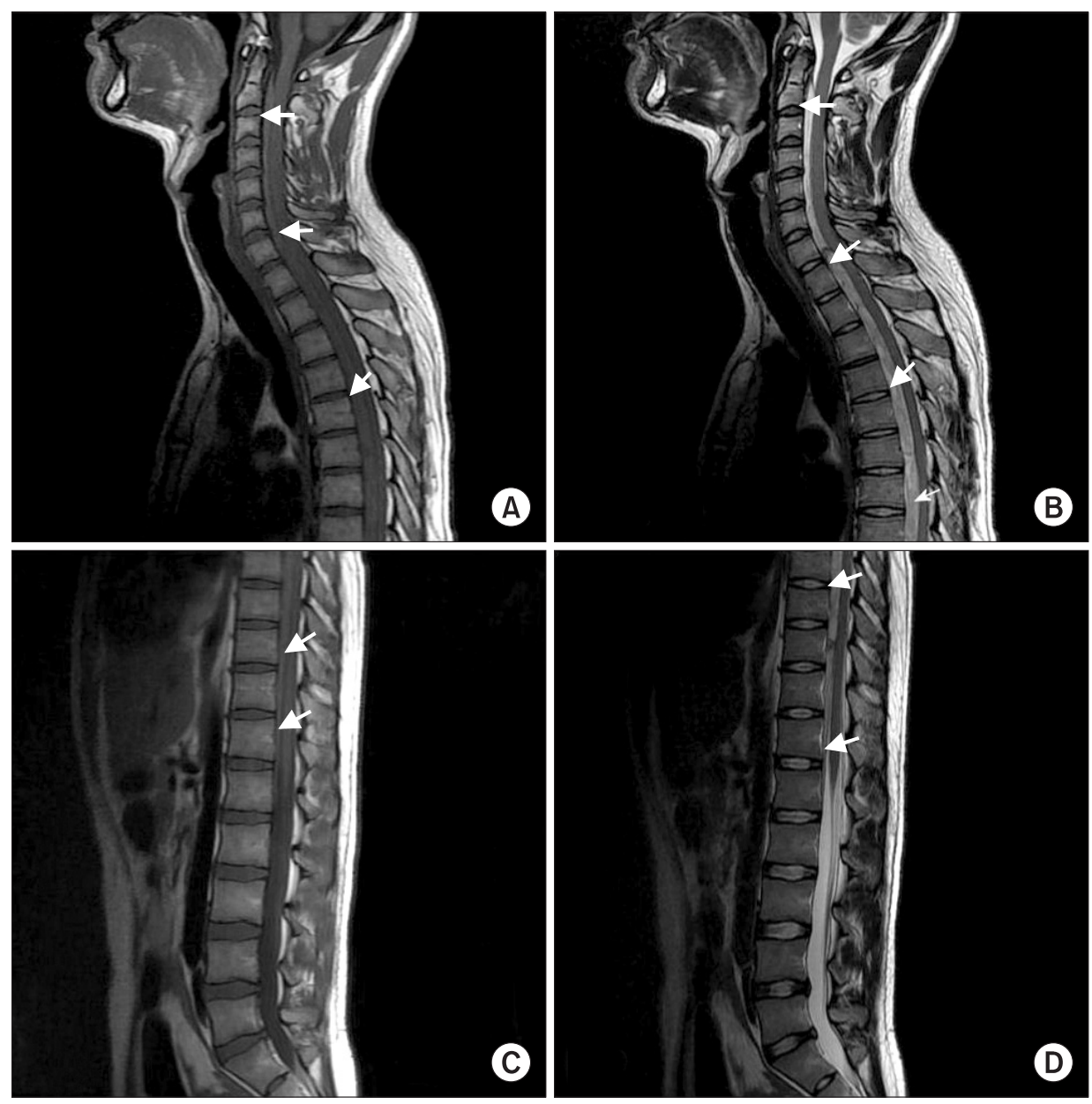

Fig. 1. Magnetic resonance imaging of the thoracic and lumbar spine, non-enhancing, margin sharp anteriorepidural hematoma with severe cord compression from T1 to L1. (A, C) Sagittal T1-weighted image of thethoracic spine shows low signal intensity from T1 to L1. (B, D) The sagittal T2-weighted image showsheterogeneous high signal intensity. 
position, and decreased with supine position. The patient was treated with hydration and absolute bed rest, and the epidural catheter was removed at 5:00 PM. On POD4 the neurologist was consulted. The impression was rule out post-dural puncture headache and conservative treatment was recommended. The patient was treated with hydration, bed rest, mefenamic acid 250 mg three times a day, oral acetaminophen 1,300 mg two times a day. However, the patient complained headache and intravenous injection of ketorolac $30 \mathrm{mg}$ was given three times within 20 hours.

At 5:20 AM on POD5, the patient complained of neck pain and right arm pain with tingling sensation. At 6:10 AM, the patient complained of weakness with numbness in both lower legs. The motor power of both lower limbs was $1 / 5$ in all muscle groups and dorsiflexion of both feet was impossible. Deep tendon reflexes were absent bilaterally. Urgent magnetic resonance imaging of the patient (Fig. 1) revealed a massive anterior epidural hematoma compressing the spinal cord from the first thoracic vertebrae to the first lumbar vertebrae. At 8: $45 \mathrm{AM}$, decompressive laminectomy and hematoma evacuation was performed from T1 to T9. At 10:30 AM, the surgeon reported coagulopathy on the operating field, but intraoperative lab findings which performed just before surgical incision were unremarkable $(\mathrm{Hb}$ : $10.8 \mathrm{mg} / \mathrm{dl}$, platelet count 266,000 / $\mathrm{mm}^{3}$, PT: 13.7 (INR: 1.23 ), aPTT: 26.9, fibrinogen 506.7). We conducted a platelet function test with PFA-100 ${ }^{\circledR}$ (Siemens, New York, USA) to rule out platelet dysfunction and found significant platelet dysfunction (collagen/epinephrine: more than 300 seconds [normal range: 61-192], collagen/Adenine di-phosphate: 124 seconds [normal range: 61-110]).

After 10 units of platelet concentrate (PC) infusion, bleeding tendency of the operation field was improved. After the end of surgery, the patient recovered uneventfully and transferred to intensive care unit. The operation time was 10 hours and 5 minutes. Therefore, we transfused ten units of PC, nine units of FFP, and five units of PRBC during the operation. Following the operation (6:50 PM), his motor function in both lower limbs was $4 / 5$ with tingling sensation. We asked the local clinic (where the patient's hemorrhoid was treated) about his medications and informed that he had been administered $10 \mathrm{mg}$ of naftazone, three times a day, for seven days. On the POD2, the patient's motor and sensory function returned to normal in both limbs. On POD3, tramadol $37.5 \mathrm{mg}$ with acetaminophen $325 \mathrm{mg}$ three times a day was started instead of ketorolac or other NSAIDs. On POD8, four days after stop taking any kinds of NSAIDs or anticoagulants, his platelet function analysis value decreased to normal range (collagen/epinephrine; $>300 \rightarrow 107$ seconds, collagen/ADP; $124 \rightarrow 70$ seconds).

On POD 13, the patient transferred to the rehabilitation department and to treat neurologic sequelae (neurogenic bladder and bowel). He was discharged on POD36 without specific complications except mild voiding difficulty.

\section{Discussion}

Known risk factors of the spinal epidural hematoma are difficult or traumatic needle and catheter insertion, ankylosing spondylitis, and anticoagulation/coagulopathy. Typical presentation consists of sharp back pain and associated motor and sensory deficit progressing to paraplegia, with a poor prognosis of recovery if decompressive laminectomy is not performed within eight hours.

This case had several risk factors of epidural hematoma. First, needle insertion was difficult and 'blood tinged cerebrospinal fluid' was noted during the first insertion which means vascular injury in spinal canal.

Second, there were several medications which can induce platelet dysfunction. Naftazone (Mediaven, 1, 2-naphthoquinone-2semicarbazone) is used to treat mild to moderate peripheral vascular diseases or venous insufficiency (varicose veins, hemorrhoids) before surgery [5]. Naftazone can increase vascular endothelial cell proliferation and inhibit platelet aggregation induced by ADP or thrombin in-vitro together with thrombin stimulated 5-HT release. Its dose is $10 \mathrm{mg}, 3$ times a day, at least 1 month. This patient had $10 \mathrm{mg}$ of naftazone, 3 times a day, for 7 days. Ex-vivo and in-vivo studies have shown that naftazone has similar inhibitory effects on rat platelet functions as ticlopidine $[5,6]$; however, there have been no reports of complications during regional anesthesia or analgesia following naftazone therapy and how long the antiplatelet effect persists after the drug is stopped.

NSAIDs generally cause transient, dose dependent and modest increases in the bleeding time, however, these prolongations often do not exceed the upper limit of the normal range for bleeding time [7]. But if NSAIDs are used with other anticoagulants (wafarin, aspirin, other NSAIDs, herbal medications), its adverse effect on coagulation can be dangerous $[3,4,7,8]$. The American Society of Regional Anesthesia has emphatically stated that the risk of epidural hematoma is very low if antiplatelet drugs are used alone without any other anticoagulants [9].

After the first surgery, the patient started to take mefenamic acid $250 \mathrm{mg}$, three times a day, for 4 days. The fourth day he had intravenous ketorolac $30 \mathrm{mg}$ three times a day and the next day he got epidural hematoma. And his abnormal PFA finding was recovered after platelet concentrate infusion and stop taking NSAIDs.

We consulted to hematologist in our hospital and the answer was drug induced platelet dysfunction. The dose of each drugs (naftazone, mefenamic acid and ketorolac) alone were not enough to make significant platelet dysfunction. However, we 
could not find any other drugs or evidence of diseases which can induce such a severe platelet dysfunction enough to make spinal epidural hematoma. We concluded that the three drugs may have additive or synergistic antiplatelet effect.

Esler et al. [10] reported a case of spinal epidural hematoma after dural puncture during combined spinal-epidural analgesia. Their patient did not complained headache, perhaps because of the "blood patch" effect of epidural hematoma. In our case, the patient was suffered with PDPH form POD2 to POD4. Thus, we can assume that clinically significant epidural hematoma of this case occurred between POD 4 and POD5. It is also possible that small bleeding caused by traumatic tap was aggravated by drugs and eventually developed into massive epidural hematoma.

Kawaguchi and Tokutomi [11] reported spinal epidural hematoma following epidural analgesia in a patient who stopped ticlopidine therapy twelve days prior to surgery who had coagulation tests and platelet counts that were within normal limits. Thus, normal PT, aPTT finding and platelet count cannot exclude the risk of coagulopathy due to platelet dysfunction.

Recently, many people are taking medications, herbal medicines or food which can decrease platelet function without awareness [4]. Taken together, these findings indicate that it is necessary to confirm that platelet function is normal before conducting neuroaxial anesthesia or analgesia in patients who have this kind of risks in addition to anticoagulation.

We tested the patient's platelet function using a PFA- $100{ }^{\circledR}$ instrument. The PFA- $100^{\circledR}$ appears to be superior to the bleeding time and is therefore recommended as a replacement screening test [12]. However, the PFA- $100^{\circledR}$ is sensitive to many variables that influence platelet function, including abnormalities in platelet number, hematocrit, drug and dietary effects, platelet receptor defects, von Willebrand's factor defect, release and granular defect. Therefore, a full blood count should always be conducted to exclude thrombocytopenia or anemia. In this case, the patient's laboratory findings were all within normal limit including Von Willebrand's factor. The patient had no family history of bleeding tendency, too.

In conclusion, we report here a case of iatrogenic epidural hematoma following traumatic needle insertion in a patient who was treated with naftazone and NSAIDs. It is important to confirm normal platelet function before and after neuroaxial anesthesia and analgesia in any patient under risk of platelet dysfunction. Multiple NSAIDs therapy is a bad choice because of its ceiling effect and increasing complications. Especially, NSAIDs treatment should be careful in a patient who is taking anticoagulants. If patients have a history of medications to treat vascular disease prior to surgery, it is important to identify the type of drugs and confirm the normal coagulation function, including platelet function. Moreover, PFA- $100^{\circledR}$ appears to be superior to the bleeding time and can be useful during operation.

\section{Acknowledgments}

This article was funded by Dankook Univeristy.

\section{References}

1. Schwarz SK, Wong CL, McDonald WN. Spontaneous recovery from a spinal epidural hematoma with atypical presentation in a nonagenarian. Can J Anaesth 2004; 51: 557-61.

2. Fox J. Spinal and epidural anesthesia and anticoagulation. Int Anesthesiol Clin 2001; 39: 51-61.

3. Galliard-Grigioni KS, Fehr M, Reinhart WH. Influence of combinations of acetylsalicylic acid, acetaminophen, and diclofenac on platelet aggregation. Eur J Pharmacol 2008; 595: 65-8.

4. Abebe W. Herbal medication: potential for adverse interactions with analgesic drugs. J Clin Pharm Ther 2002; 27: 391-401.

5. Durand P, Bloy C, Peltier-Pujol F, Blache D. In-vitro and ex-vivo inhibition of blood platelet aggregation by naftazone. J Pharm Pharmacol 1996; 48: 566-72.

6. McGregor L, Chignier E, Bloy C, Rousselle C, Peltier-Pujol F, McGregor JL. Effect of naftazone on in vivo platelet function in the rat. Platelets 1999; 10: 66-70.

7. Schafer Al. Effects of nonsteroidal anti-inflammatory drugs on platelet function and systemic hemostasis. J Clin Pharmacol 1995; 35: 20919.

8. Kaneda T, Urimoto G, Suzuki T. Spinal epidural hematoma following epidural catheter removal during antiplatelet therapy with cilostazol. J Anesth 2008; 22: 290-3.

9. Horlocker TT, Wedel DJ, Rowlingson JC, Enneking FK, Kopp SL, Benzon HT, et al. Regional anesthesia in the patient receiving antithrombotic or thrombolytic therapy: American Society of Regional Anesthesia and Pain Medicine Evidence-Based Guidelines (Third Edition). Reg Anesth Pain Med 2010; 35: 64-101.

10. Esler MD, Durbridge J, Kirby S. Epidural haematoma after dural puncture in a parturient with neurofibromatosis. Br J Anaesth 2001; 87: 932-4.

11. Kawaguchi S, Tokutomi S. A case of epidural hematoma associated with epidural catheterization which occurred on 12th days after the last medication of ticlopidine hydrochloride. Masui 2002; 51: 526-8.

12. Harrison P. Platelet function analysis. Blood Rev 2005; 19: 111-23. 